\title{
Effect of Boussinesq-Stokes Suspension over an Exponentially Stretching Sheet in a Hydromagnetic Flow
}

\author{
S Manjunath* and L Venkata Reddy ${ }^{\dagger}$
}

\begin{abstract}
The paper presents the study of velocity profiles in a hydromagnetic flow of Boussinesq-Stokes suspension over an exponentially stretching impermeable sheet. The basic equations governing the flow are in the form of partial differential equations. The equations have been transformed to nonlinear ordinary differential equation by applying a suitable local similarity transformation. The solution of the transformed equation is obtained by using differential transform method (DTM) with assistance from the Newton-Raphson method in obtaining the unknown initial values. The solution is obtained as a power series with assured convergence. The effects of local Chandrasekhar number and couple stress parameter on velocity profiles are studied. The findings of the study are represented graphically.
\end{abstract}

Keywords: MHD, Boussinesq-Stokes suspension, Exponential stretching Sheet, Couple stress Chandrasekhar Number, Differential Transform Method.

\footnotetext{
* Department of Mathematics, B. N. M. Institute Technology, 12th Main, 27th Cross, Banashankari 2nd Stage, Bangalore - 560 070, Karnataka, India, adrmanjus@gmail.com

t Department of Mathematics, B. N. M. Institute Technology, 12th Main, 27th Cross, Banashankari 2nd Stage, Bangalore - 560 070, Karnataka, India, lakshmaiahreddy@yahoo.com
} 


\section{Introduction}

Boundary layer flow over a continuously stretching sheet has several applications such as hot rolling, wire drawing, glass fiber production and paper production. After the pioneering theoretical work of Crane [1], innumerable works have appeared on various aspects of flow characteristics in Newtonian fluids' boundary layer flow over a stretching boundary. Most of these works deal with the study of boundary layer flows over a stretching surface where the velocity of the surface is assumed to stretch in linear proportion to the distance from the fixed origin (slit) as pioneered by Crane [1] or in quadratic proportion as pioneered by Kumaran and Ramanaiah [2].

The liquids surrounding the stretching sheet are known to serve the purpose of cooling of the sheet at a desired rate and it is common knowledge now that this has a great influence on the characteristics of the final product. A good discussion on this is given in the work of Siddheshwar and Mahabaleshwar [3]. Magnetic field is known to regulate the flows of electrically conducting liquids and this can be used to good effect in the applications involving the stretching sheet. Abel and Begum [4] carried out an analysis to study the magneto hydrodynamic boundary layer flow behavior and heat transfer characteristics of a viscoelastic fluid flow over a stretching sheet with radiation and for the case of large Prandtl numbers. Khaleque and Samad [5] analyzed the radiation and viscous dissipation effects on a steady two-dimensional magneto-hydrodynamics free convection flow along a stretching sheet with heat generation. From the practical point of view, stretching cannot be a linear function or even a quadratic function of the axial coordinate unless the process is precisely done.

In view of this, there is a need to make investigation of flow due to stretching that is exponential in the axial coordinate. Magyar and Keller [6] for first time studied the heat and mass transfer in a boundary layer flow due to an exponentially stretching continuous surface. Elbashbeshy [7] studied the exponential stretching sheet problem with suction. Partha et al. [8] examined the effect of viscous dissipation on the mixed convective heat transfer of a 94 
Newtonian liquid over an exponentially stretching surface. Khan and Sanjayanand [9] extended this study to viscoelastic liquids. Sanjayanand and Khan [10] studied heat and mass transfer in a viscoelastic boundary layer flow over exponentially stretching sheet. Chen et al. [11] used the Adomian method for studying the momentum and heat transfer equation in a Newtonian liquid with exponential surface conditions. Sajid and Hayat investigated [12] the influence of thermal radiation on the boundary layer flow due to exponentially stretching sheet. Haas and Oliveski [13] studied the exponential class of similarity solutions for the hydromagnetic Falkner-Skan equation.

Most of the above works use either a first order solution or a difficult- to - use homotopy analysis method. We have chosen to study the magnetohydrodynamic stretching sheet problem involving a Boussinesq-Stokes suspension on the reason explained earlier. Motivated by the above investigations and possible applications, authors contemplate to study momentum in a flow of Boussinesq - Stokes suspension over an exponentially stretching sheet. The differential transform method (DTM) is used to obtain a series solution.

\section{Nomenclature}

C Couple stress parameter

$f \quad$ Dimensionless stream function

$l \quad$ Reference length

Q Chandrasekhar number

Re Local Reynolds number

u Horizontal velocity component

v Vertical velocity component

x Horizontal Cartesian coordinate

y Vertical Cartesian coordinate 


\section{Symbols}

$\eta \quad$ Similarity variable

$v \quad$ Kinematics coefficient of viscosity

$v^{\prime} \quad$ Couple stress viscosity

$\psi \quad$ Stream function

$\sigma \quad$ Electrical conductivity

$\rho \quad$ Density

\section{Mathematical formulation}

The governing boundary layer equations for momentum in the present problem are

$$
\begin{gathered}
\frac{\partial u}{\partial x}+\frac{\partial v}{\partial y}=0 \\
u \frac{\partial u}{\partial x}+v \frac{\partial v}{\partial y}=v \frac{\partial^{2} u}{\partial y^{2}}-\left(\frac{\mu_{m}^{2} \sigma H_{0}^{2}}{\rho}\right) u-v^{\prime} \frac{\partial^{4} u}{\partial y^{4}}
\end{gathered}
$$

where $\mathrm{u}$ and $\mathrm{v}$ are the velocity components of the fluid in $\mathrm{x}$ and $\mathrm{y}$ directions respectively, $v$ is the kinematics coefficient of viscosity, $v^{\prime}$ is couple stress viscosity, $\mu_{m}$ is the magnetic permeability. $\sigma$ is the electrical conductivity, $H_{0}$ is the applied magnetic field and $\rho$ is the density of the fluid.

The flow, as mentioned earlier, is considered to be generated solely by stretching the boundary surface in the $x$ - direction with the stretching assumed to be in exponential proportion to the axial coordinate. Following Elbashbeshy [7], we employ the following boundary conditions on velocity. 


$$
\left.\begin{array}{l}
u=U_{w}(x)=U_{0} \exp \left(\frac{x}{l}\right), v=0, \frac{\partial^{2} u}{\partial y^{2}}=0 \text { at } \mathrm{y}=0 \\
u \rightarrow 0, \frac{\partial^{2} \mathrm{u}}{\partial \mathrm{y}^{2}} \rightarrow 0 \text { as } y \rightarrow \infty
\end{array}\right\}
$$

where $U_{w}$ stands for stretching velocity of the boundary, $U_{0}$ is a constant and $l$ is the reference length. In the next section, we present a local similarity solution of the boundary value problem given by the equations (1) - (3).

\section{Solution of the momentum equation}

Introducing the stream function $\psi(\mathrm{x}, \mathrm{y})$ defined by

$$
\mathrm{u}=\frac{\partial \psi}{\partial \mathrm{y}}, \mathrm{v}=-\frac{\partial \psi}{\partial \mathrm{x}}
$$

into equation (2) we get

$$
\begin{aligned}
v^{\prime} \frac{\partial^{5} \psi}{\partial y^{5}}- & v \frac{\partial^{3} \psi}{\partial y^{3}}+\frac{\partial \psi}{\partial y} \frac{\partial^{2} \psi}{\partial x \partial y} \\
& -\frac{\partial \psi}{\partial x} \frac{\partial^{2} \psi}{\partial y^{2}}+\left(\frac{\mu_{m}^{2} \sigma H_{0}^{2}}{\rho}\right) \frac{\partial \psi}{\partial y}=0
\end{aligned}
$$

The boundary conditions for solving equation (5) can be obtained from equations (3) as

$$
\left.\begin{array}{l}
\frac{\partial \psi}{\partial \mathrm{y}}=\mathrm{U}_{0} \exp \left(\frac{x}{l}\right), \frac{\partial \psi}{\partial \mathrm{x}}=0, \frac{\partial^{3} \psi}{\partial \mathrm{y}^{3}}=0 \text { at } \mathrm{y}=0 \\
\frac{\partial \psi}{\partial \mathrm{y}} \rightarrow 0, \frac{\partial^{3} \psi}{\partial \mathrm{y}^{3}} \rightarrow 0 \text { as } y \rightarrow \infty
\end{array}\right\}
$$


We now use the following non-dimensionalization and transformation:

$$
\mathrm{X}=\frac{x}{l}, \mathrm{Y}=\frac{\mathrm{y}}{l}, \Psi(X, Y)=\frac{\psi(\mathrm{x}, \mathrm{y})}{\mathrm{v}}=\sqrt{2 \operatorname{Re}} f(\eta) \exp \left(\frac{X}{2}\right)
$$

where $\eta=Y \sqrt{\frac{\operatorname{Re}}{2}} \exp \left(\frac{X}{2}\right)$ is similarity variable. Substituting the above transformation into equations (5) and (6), we obtain the nonlinear boundary value problem given below:

$$
\left.\begin{array}{c}
C f_{\eta \eta \eta \eta \eta}-f_{\eta \eta \eta}+2 f_{\eta}^{2}-f f_{\eta \eta}+2 Q f_{\eta}=0, \\
f=0, f_{\eta}=1, f_{\eta \eta \eta}=0 \text { at } \eta=0 \\
f_{\eta} \rightarrow 0, f_{\eta \eta \eta} \rightarrow 0 \text { as } \eta \rightarrow \infty
\end{array}\right\},
$$

and also we have assumed the following conditions to solve the fifth order differential equation (8)

$$
f_{\eta \eta}=\alpha, f_{\eta \eta \eta \eta}=\beta \text { at } \eta=0 \text {, }
$$

where $Q=\left(\frac{\mu_{m}^{2} l \sigma H_{0}^{2}}{\rho U_{w}}\right)$ is the dimensionless local Chandrasekhar number, $\quad C=\left(\frac{v^{\prime} \exp (2 X)}{2 l^{3} U_{w}}\right)$ is local couple stress parameter and $U_{w}=U_{0} \exp (X)$. In order to use the differential transform method for solving the equations (8), we use a new independent variable by defining $Z=1-e^{-\eta}$. Equation (8) now becomes 


$$
\begin{aligned}
& C f^{\prime \prime \prime \prime \prime}-10 C f^{\prime \prime \prime \prime}-(1-25 C) f^{\prime \prime \prime}-(15 C-3) f^{\prime \prime}+10 C\left(3 Z-3 Z^{2}+Z^{3}\right) f^{\prime \prime \prime \prime}- \\
& (1-C-2 Q) f^{\prime}-C\left(4 Z-6 Z^{2}+4 Z^{3}-Z^{4}\right) f^{\prime \prime \prime \prime \prime}+(1-25 C)\left(2 Z-Z^{2}\right) f^{\prime \prime \prime}- \\
& (3-15 C) Z f^{\prime \prime}-f f^{\prime \prime}+2\left(f^{\prime}\right)^{2}+f f^{\prime}+Z f f^{\prime \prime}-2 Z\left(f^{\prime}\right)^{2}=0 .
\end{aligned}
$$

The boundary conditions (9) and (10) in terms of $Z$ are

$$
\left.\begin{array}{l}
f=0, f^{\prime}=1, f^{\prime \prime}=\alpha+1, f^{\prime \prime \prime}=3 \alpha+2, f^{\prime \prime \prime \prime}=11 \alpha+\beta+6 \text { at } Z=0 \\
f^{\prime}=0, f^{\prime \prime \prime}=0 \text { at } Z=1
\end{array}\right\}
$$

The differential transform of $f, D^{k}[f(Z)]$ is defined as follows:

$$
F[k]=D^{k}\{f(Z)\}=\left[\frac{1}{k !} \frac{d^{k} f(Z)}{d Z^{k}}\right]_{Z=0}
$$

Applying differential transform method to equation (11), we get

$$
\begin{aligned}
C & (k+1)(k+2)(k+3)(k+4)(k+5) F[k+5] \\
= & 10 C(k+1)(k+2)(k+3)(k+4) F[k+4]+ \\
& (1-25)(k+1)(k+2)(k+3) F[k+3]+(15 C-3)(k+1)(k+2) F[k+2] \\
& +(1-C-2 Q)(k+1) F[k+1]+\sum_{r=0}^{k}(r+1)(r+2) F[r+2] F[k-r] \\
& -2 \sum_{r=0}^{k}(r+1)(k-r+1) F[r+1] F[k-r+1]- \\
& \sum_{r=0}^{k}(r+1) F[r+1] F[k-r]+(3-15 C) \sum_{r=0}^{k} \delta(k-r-1)(r+1)(r+2) F[r+2]+ \\
& C \sum_{r=0}^{k}(4 \delta(k-r-1)-6 \delta(k-r-2)+4 \delta(k-r-3)-\delta(k-r-4)) \times \\
& (r+1)(r+2)(r+3)(r+4)(r+5) F[r+5]- \\
& (1-25 C) \sum_{r=0}^{k}(2 \delta(k-r-1)-\delta(k-r-2))(r+1)(r+2)(r+3) F[r+3]- \\
& 2 \sum_{k_{2}}^{k} \sum_{k_{1}}^{k_{2}}\left(k_{1}+1\right)\left(k_{2}-k_{1}+2\right) F\left[k_{1}+1\right] F\left[k_{2}-k_{1}+1\right] \delta\left(k-k_{2}-1\right) \\
& 10 C \sum_{r=0}^{k}(3 \delta(k-r-1)-3 \delta(k-r-2)+\delta(k-r-3))(r+1)(r+2)(k+3)(k+4) F[r+4]- \\
& \sum_{k_{1}}^{k_{2}}\left(k_{1}+1\right)\left(k_{2}+2\right) F\left[k_{1}+2\right] F\left[k_{2}-k_{1}\right] \delta\left(k-k_{2}-1\right)+ \\
& \\
&
\end{aligned}
$$


where $k=0,1,2,3, \ldots$

Using Mathematica, we can obtain $F[5], F[6], F[7], \ldots$ from equation (14) with

$$
\begin{gathered}
F[0]=0, F[1]=1, F[2]=\left(\frac{\alpha+1}{2}\right), F[3]=\left(\frac{\alpha}{2}+\frac{1}{2}\right) \text { and } \\
F[4]=\left(\frac{11 \alpha}{24}+\frac{\beta}{24}+\frac{1}{4}\right) .
\end{gathered}
$$

Thus, we have

$$
\begin{gathered}
F[5]=\left(\frac{1}{5}+\frac{5 \alpha}{12}+\frac{\beta}{12}-\frac{Q}{60 C}-\frac{1}{60 C}\right), \\
F[6]=\left(\frac{1}{6}+\frac{137 \alpha}{360}+\frac{17 \beta}{144}-\frac{Q}{24 C}-\frac{1}{24 C}-\frac{\alpha Q}{360 C}-\frac{\alpha}{240 C}+\frac{\beta}{720 C}\right), \\
F[7]=\left(\begin{array}{l}
\left.\frac{1}{7}+\frac{7 \alpha}{20}+\frac{7 \beta}{48}-\frac{1}{2520 C^{2}}-\frac{5}{72 C}-\frac{\alpha}{80 C}-\frac{\alpha^{2}}{1680 C}\right), \\
+\frac{\beta}{240 C}-\frac{Q}{2520 C^{2}}-\frac{5 Q}{72 C}-\frac{Q \alpha}{120 C}
\end{array}\right) \\
F[8]=\left(\begin{array}{l}
\frac{1}{8}+\frac{363 \alpha}{1120}+\frac{967 \beta}{5760}-\frac{1}{720 C^{2}}-\frac{\alpha}{13440 C^{2}}+\frac{\beta}{40320 C^{2}}-\frac{7}{72 C} \\
-\frac{23 \alpha}{960 C}-\frac{\alpha^{2}}{480 C}+\frac{107 \beta}{13440 C}+\frac{Q}{720 C^{2}}-\frac{7 Q}{72 C}-\frac{Q \alpha}{20160 C^{2}} \\
-\frac{23 Q \alpha}{1440 C}-\frac{Q \beta}{20160 C}
\end{array}\right)
\end{gathered}
$$




$$
F[9]=\left(\begin{array}{l}
\frac{1}{9}+\frac{761 \alpha}{2520}+\frac{89 \beta}{480}-\frac{1}{181440 C^{3}}-\frac{13}{4320 C^{2}}-\frac{\alpha}{3360 C^{2}} \\
-\frac{\alpha^{2}}{120960 C^{2}}+\frac{\beta}{10080 C^{2}}-\frac{1069}{8640 C}-\frac{3 \alpha}{80 C}-\frac{13 \alpha^{2}}{2880 C} \\
+\frac{25 \beta}{2016 C}-\frac{\alpha \beta}{40320 C}-\frac{Q}{181440 C^{3}}-\frac{17 Q}{5670 C^{2}}-\frac{1069 Q}{8640 C} \\
+\frac{Q^{2}}{90720 C^{2}}-\frac{Q \alpha}{40 C}-\frac{Q \alpha}{5040 C^{2}}-\frac{Q \beta}{5040 C}
\end{array}\right)
$$$$
F[10]=\left(\begin{array}{l}
\frac{1}{10}+\frac{7129 \alpha}{25200}+\frac{4523 \beta}{22680}-\frac{1}{40320 C^{3}}-\frac{\alpha}{1209600 C^{3}} \\
+\frac{\beta}{3628800 C^{3}}-\frac{1}{192 C^{2}}-\frac{173 \alpha}{241920 C^{2}}-\frac{Q}{40320 C^{3}} \\
-\frac{13 Q}{2520 C^{2}}-\frac{19 Q}{128 C}+\frac{Q^{2}}{20160 C^{2}}-\frac{Q \alpha}{1814400 C^{3}} \\
-\frac{859 Q \alpha}{1814400 C^{2}}-\frac{3013 Q \alpha}{86400 C}+\frac{Q^{2} \alpha}{907200 C^{2}}-\frac{Q \beta}{907200 C^{2}} \\
-\frac{29 Q \beta}{60480 C}-\frac{\alpha^{2}}{26880 C^{2}}+\frac{29 \beta}{1120960 C^{2}}-\frac{19}{128 C} \\
-\frac{3013 \alpha}{57600 C}-\frac{\alpha^{2}}{128 C}+\frac{20801 \beta}{1209600 C}-\frac{\alpha \beta}{8960 C}
\end{array}\right)
$$

Taking the inverse differential transform, we get

$$
\begin{aligned}
f(Z)= & F[0]+F[1] Z+F[2] Z^{2}+F[3] Z^{3}+F[4] Z^{4} \\
& +F[5] Z^{5}+F[6] Z^{6}+\ldots
\end{aligned}
$$

Differentiating w.r.t. $\mathrm{z}$

$$
\begin{aligned}
f^{\prime}(Z)= & F[1]+2 F[2] Z+3 F[3] Z^{2}+4 F[4] Z^{3} \\
& +5 F[5] Z^{4}+6 F[6] Z^{5}+\ldots
\end{aligned}
$$


where $\alpha$ and $\beta$ are determined using the unused conditions $f^{\prime}(1)=0$ and $f^{\prime \prime \prime}(1)=0$ by Newton-Raphson method as shown in the table 1 .

Table 1:

\begin{tabular}{|c||c||c|c||c|c|c|}
\hline \hline Q & \multicolumn{3}{|c|}{0.00} & \multicolumn{3}{c|}{2.00} \\
\hline \hline $\mathrm{C}$ & 0.10 & 0.20 & 0.30 & 0.10 & 0.20 & 0.30 \\
\hline \hline$\alpha$ & -0.9953 & -0.9254 & -0.8866 & -1.4877 & -1.3093 & -1.2035 \\
\hline \hline$\beta$ & 4.2800 & 2.7827 & 2.1696 & 11.6072 & 7.3352 & 5.5649 \\
\hline \hline
\end{tabular}

\section{Results and discussion}

In the paper we have investigated the hydromagnetic boundary layer flow of a Boussinesq-Stokes suspension over an exponentially stretching impermeable sheet. Highly nonlinear partial differential equations characterize the flow phenomena. The equations have been transformed into non-linear ordinary differential equations by applying a suitable local similarity transformation. The solution of transformed momentum equation is obtained by using differential transform method (DTM) with assistance from the Newton Raphson in obtaining the unknown initial values. The findings of the study are depicted in the form of graphs. In figure 1, the graphs of $f(\eta)$ and $f^{\prime}(\eta)$ versus $\eta$ are drawn for different values of the couple stress parameter $C$ with local Chandrasekhar numbers $Q=$ 0.00 and $Q=2.00$. The reason for obtaining the above results is that the suspended particle in the Boussinesq - Stokes suspension increase the velocity of the carrier liquid and hence to decrease the flow. The effect of magnetic field is to oppose any motion in the liquid. On the above reason we find from figure 1 that the effect of 102 
increasing the Chandrasekhar number $\mathrm{Q}$ is to decrease the velocity profiles $f(\eta)$ and $f^{\prime}(\eta)$ through the boundary layer. Also we find from the graph that the effect of increasing the values of couple stress parameter $\mathrm{C}$ is to increase the velocity profile $f(\eta)$ and $f^{\prime}(\eta)$ through the boundary layer.

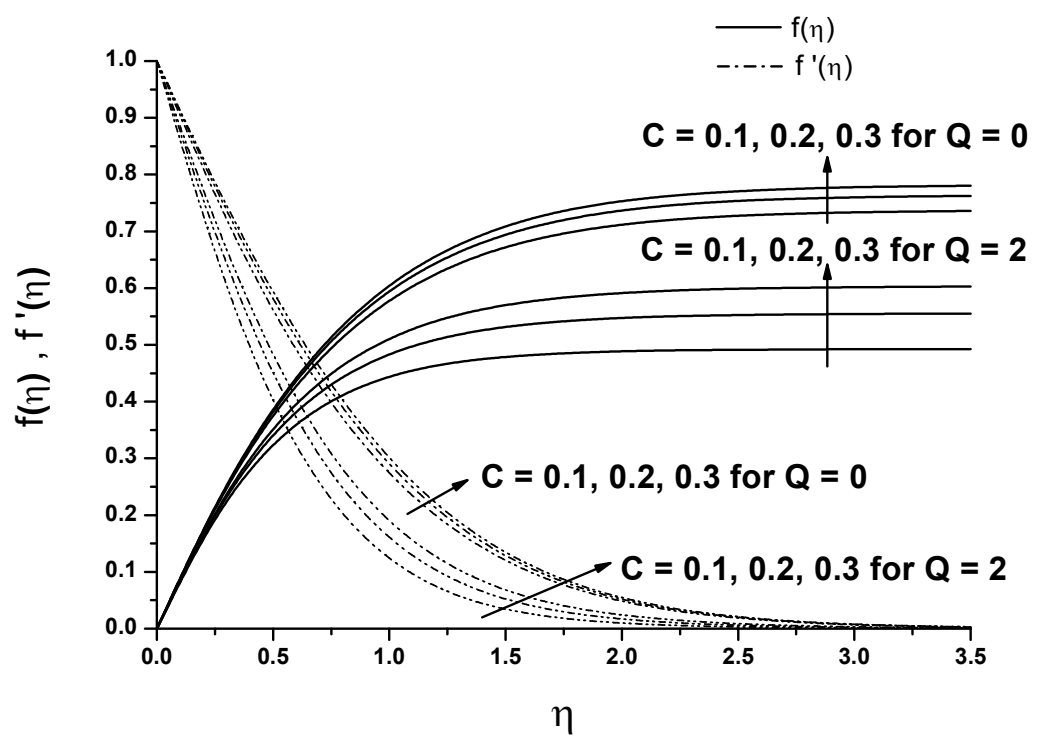

Figure 1: Plots of $f(\eta)$ and $f^{\prime}(\eta)$ verses $\eta$ for different values of $\mathrm{C}$ with $\mathrm{Q}=0.00$ and $\mathrm{Q}=2.00$.

\section{Acknowledgement}

The work reported in the paper is carried out at the V. T. U. research center in the Department of Mathematics, B. N. M. Institute of Technology, and Bangalore. The work of the authors was supported and encouraged by Dr. Pradeep G. Siddheshwar, Professor of Mathematics, Bangalore University, Bangalore, the Management, the Director, the Dean and the Principal of B. N. M. Institute of Technology, Bangalore, India. 


\section{References}

[1] L J Crane, Fast flow stretching plate, J. Appl. Math. Phys.(ZAMP), vol. 21, pp. 645-647, 1970.

[2] V Kumaran and G Ramanaiah, A note on the flow over a stretching sheet, Acta Mech., vol. 116, pp. 229-233, 1996.

[3] P G Siddheshwar and U S Mahabaleswar, Effects of radiation and heat source on MHD flow of a viscoelastic liquid and heat transfer over a stretching sheet, Int. J. of Non-linear mechanics, vol. 40, pp. 807-820, 2005.

[4] M Subhas and Gousia Begum, Heat transfer in MHD viscoelastic fluid flow on stretching sheet with heat source/sink, viscous dissipation, stress work and radiation for the case of large Prandtl number, Chemical Engineering Communication, vol. 12, pp. 1503-1523, 2008.

[5] S K Tania, M A Khaleque and Samad, Effects of radiation, heat generation and viscous dissipation on MHD free convection flow along a stretching, Research Journal of Applied Sciences, Engineering and Technology, vol. 24, pp. 368-377, 2010.

[6] E Magyari and B Keller, Heat and mass transfer in the boundary layer on an exponential continuous surface, J. Phys. D: Appl. Phys., vol. 32, p. 577, 1999.

[7] E M A Elbashbeshy, Heat transfer over an exponentially stretching continuous surface with suction, Arch. Mech., vol. 53, pp. 643-651, 2001.

[8] M K Partha, P V S N Murthy and G P Rajasekhar, Effect of viscous dissipation on the mixed convection heat transfer from an exponentially stretching surface, Heat and Mass Tranfer, vol. 41, pp. 360-366, 2005.

[9] Sujit Kumar Khan and Emmanuel Sanjayanand, Viscoelastic boundary layer flow and heat transfer over an exponential stretching sheet, Int. J. of Heat and Mass Transfer, vol. 48, pp. 1534-1542, 2005.

[10] Emmanuel Sanjayanand and Sujit Kumar Khan, On heat and mass transfer in a viscoelastic boundary layer flow over an exponentially stretching sheet, Int. J. Thermal Sci., vol. 4, pp. 819-828, 2006.

[11] Xuehui Chen, Liancum Zheng and Xinxin Zhang, An efficient analytical decomposition and numerical procedure for boundary layer flow on a continuous stretching surface, J. University of Science and Technology Beijing, vol. 13, p. 401, 2006.

[12] M Sajid and T Hayat, Influence of thermal radiation on the boundary layer flow due to an exponentially stretching sheet, Int. Comm. Heat and Mass Transfer, vol. 35, pp. 347-356, 2008.

[13] F Haas and R C Oliveski, Exponential class of similarity solutions for the hydromagnetic Falkner-skan equations, Phys. Fluid Dyn., 2011. 